\title{
The Influence of Depression and School Life on the Quality of Life of Korean Child and Adolescent Patients with Attention-Deficit/Hyperactivity Disorder: A Comparison of the Perspectives of the Patients and Their Caregivers
}

\author{
Byeong-Eon Park, Jeong-Seop Lee, Hee-Yun Kim, Jae-Nam Bae, Won-Hyoung Kim, \\ Hye-Young Kim, Mi-Roo Rim, Sang-Gu Kang, and Seo-Hyun Choi \\ Department of Psychiatry, University of Inha College of Medicine, Inha Medical Center, Incheon, Korea
}

\begin{abstract}
Objectives: This study aimed to compare the quality of life reported by patients with attention-deficit/hyperactivity disorder (ADHD) to the patients' quality of life as reported by their caregivers. In addition, it aimed to examine how emotional problems, including depression and anxiety, and the severity of the symptoms affect the quality of life reported by the patients and their caregivers.

Methods: The patients' quality of life and their degree of depression and anxiety were measured using the Pediatric Quality of Life Inventory (PedsQL) 4.0 Child Self-Report, the Children's Depression Inventory (CDI), and the Revised Children's Manifest Anxiety Scale, respectively. The caregivers' perception of the patients' quality of life and severity of the ADHD symptoms were measured using the PedsQL 4.0 Parent Proxy Report and the Conners' Parent Rating Scale (CPRS), respectively. A total of 66 participants completed the survey. The independent-samples t-test, Pearson's correlation analysis, and multiple regression analysis were conducted.

Results: The mean score of the PedsQL 4.0 Child Self-Report was significantly higher than the mean score of the PedsQL 4.0 Parent Proxy Report. However, for school function, the PedsQL 4.0 Child Self-Report score was significantly lower than that of Parent Proxy Report. The correlation between the PedsQL 4.0 Child Self-Report and PedsQL 4.0 Parent Proxy Report scores was significant only for emotional function and social function. The multiple regression analysis showed that the PedsQL 4.0 Child Self-Report and PedsQL 4.0 Parent Proxy Report scores were significantly predicted by the CDI and CPRS scores, respectively.

Conclusion: Our results demonstrate that there are clear differences between the quality of life reported by the patient themselves and that reported by their caregivers. In addition, the findings suggest that it is critical to treat the patients' accompanying depressive symptoms.
\end{abstract}

Key Words: Attention-deficit/hyperactivity disorder; Quality of life; Depression; Anxiety; School function.

Received: August 16, 2018 / Revision: August 30, 2018 / Accepted: September 3, 2018

Address for correspondence: Jeong-Seop Lee, Department of Psychiatry, University of Inha College of Medicine, Inha Medical Center, 27 Inhang-ro, Jung-gu, Incheon 22332, Korea

Tel: +82-32-890-3477, Fax: +82-32-890-3536, E-mail: soulfree@inha.ac.kr

\section{INTRODUCTION}

Recently, in clinical studies of attention-deficit/hyperactivity disorder (ADHD), quality of life has been emphasized as a key factor in the success or failure of treatment $[1,2]$. Patients with ADHD cannot focus on daily matters and forget what they should do, are not able to complete their own plans, and their academic achievements are quite low. Their hyperactivity and impulsivity can also lead to a deterioration

This is an Open Access article distributed under the terms of the Creative Commons Attribution Non-Commercial License (https://creativecommons.org/licenses/by-nc/4.0) which permits unrestricted non-commercial use, distribution, and reproduction in any medium, provided the original work is properly cited. in their relationships with colleagues [3]. In addition, ADHD can have a negative influence on various aspects of their life, including self-respect, social functions, and caregiver-andchild relationships [4]. Due to these, it is necessary to review the quality of life of patients with ADHD.

Quality of life is generally known as a multi-dimensional concept, and its core areas include physical, psychological, cognitive, and social functions [2]. These areas of quality of life can be affected by a variety of factors, and exploring what kind of factors are related to the quality of life of ADHD patients is helpful for setting the direction of the treatment and predicting a prognosis [5]. In general, it is known that the 
lowered quality of life of patients with ADHD is linked to the severity of the symptoms, comorbidities, emotional problems, and psycho-social stress [6-9]. However, studies of the influence of these factors on quality of life or comparisons are rare. Moreover, there have been few domestic studies of these factors.

In the recent studies of quality of life, the evaluator's role has been emphasized because of its subjectivity, and according to these studies, when the quality of life is evaluated by the caregivers, only one aspect that affects the quality of life of the patients is shown [10]. In addition, the studies in which the quality of life is assessed by the caregivers do not include questions about the patients' own perspective of their quality of life, which leads to lower validity [10]. The reason is that while there is some agreement between the measurements of quality of life by the patients and by the caregivers, there are differences in areas that are inconspicuous, such as emotional or social functions $[11,12]$. However, until now, most of the studies of the quality of life of children and adolescents have not included an evaluation of quality of life by the patients themselves [2].

Therefore, this study aimed to directly ask the patients about their quality of life and to compare it with their quality of life as perceived by their caregivers. In addition, it aimed to examine how emotional problems, including depression and anxiety, and the severity of the symptoms affect the quality of life as reported by the patients and caregivers, respectively.

\section{METHODS}

\section{Subjects}

Among the patients in the outpatient setting of a university hospital's Department of Psychiatry, those diagnosed with ADHD (based on the fifth edition of the Diagnostic and Statistical Manual of Mental Disorders' criteria) were recruited. The study focused on the patients in the outpatient setting of the Department of Psychiatry and their main caregivers between January 1, 2017 and February 28, 2018.

Patients with ADHD ranging in age between 8 and 17 years were recruited for the study, and those who had been hospitalized due to spastic diseases, organic brain diseases, or other internal diseases within the past 4 weeks were excluded. The main caregiver was defined as the family member who spends the most time with the patient.

After explaining the purpose of the study to the patients who fit the criteria and to their main caregivers during the recruitment period, their respective consent to participate in the study was provided in a document. Both the patients and their caregivers who gave their consent completed their questionnaire responses with help from medical personnel in different places. This study was approved by the Institution- al Review Board of Inha University Hospital, and all of the patients and their caregivers gave their written consent (IRB No. 2016-12-001).

\section{Procedure}

The questionnaire was completed when the patients and their caregivers visited the outpatient setting. This was done once per patient and caregiver. If any refused, they were excluded.

\section{Measures}

\section{Korean version of the Pediatric Quality of Life Inventory} (PedsQL) 4.0

\section{PedsQL 4.0 Child Self-Report}

To evaluate the quality of life of the patients, the Korean edition of the self-report version of the Pediatric Quality of Life Inventory version 4.0 Generic Core Scales (PedsQL) was used, which is classified into a children's version (age 8-12 years) and teenagers' version (age 13-18 years). It was translated from the general version of the quality of life test for children that was created by Varni, Seid, and Kurtin and validated by Kook and Varni [13]. It has a total of 23 questions that cover eight physical areas, five emotional areas, five social areas, and five school areas. Each question is responded to on a scale ranging from 0 to $4(0=$ no problem, $1=$ barely a problem, $2=$ sometimes a problem, $3=$ frequently a problem, and $4=$ almost always a problem). Each question is reverse scored and 0 points is converted to 0,1 point is converted to 25,2 points are converted to 50,3 points are converted to 75 , and 4 points are converted to 100 . After summing the points for each area and obtaining the total score, it is divided by the total number of questions and a score between 0 and 100 is obtained. When the calculated score is higher, it is interpreted as the child's quality of life being higher. In the study by Kook and Varni [13], the Cronbach's $\alpha$ ranged between 0.72 and 0.87 , and the internal consistency was high at 0.90 .

\section{PedsQL 4.0 Parent Proxy Report}

To evaluate the quality of life of the patients from the caregivers' perspective, the Korean edition of the parental version of the PedsQL 4.0 Generic Core Scales was used, which is classified into a parents of children version (age 8-12 years) and a parents of teenagers version (age 13-18 years). It was also translated from the general type of quality of life test for children that was created by Varni, Seid, and Kurtin and validated by Kook and Varni [13]. It contains a total of 23 questions that cover eight physical areas, five emotional areas, five social areas, and five school areas. In the study by 
Kook and Varni [13], the Cronbach's $\alpha$ ranged between 0.75 and 0.88 , and the internal consistency was high at 0.90 .

\section{Conners' Parent Rating Scale (CPRS)}

To assess the severity of the patients' ADHD symptoms, the Korean version of the Conners' Parent Rating Scale (CPRS) was used. This scale is used in clinical settings and research studies to evaluate ADHD symptoms and the related problematic behaviors of children with ADHD between the ages of 3 and 17 years. There are two versions: the basic version (containing 93 questions) and the simple version (containing 48 questions). The simple version, which was developed after careful consideration of various psychometric evidence accumulated from the basic version, obtains more concise information about the children's problematic behaviors. It was translated and validated by Park et al. [14], and it consists of a total of 48 questions. The severity of the behaviors is reported using a scale ranging from 0 to 3 points. In the parents' version, there are five sub-scales that measure attitude problems, learning problems, psycho-physical problems, impulsivityhyperactivity problems, and anxiety problems. In addition, an index for hyperactivity is included. In the study by Park et al. [14] the Cronbach's $\alpha$ for the sub-factors ranged from 0.53 to 0.82 .

\section{Children's Depression Inventory (CDI)}

To measure the patients' depression, the Children's Depression Inventory (CDI) was used. It was developed by Kovasc and has been translated and adopted by Cho and Lee [15]. The CDI consists of 27 questions that can be used with children and adolescents up to 17 years of age. It is a self-report instrument, and it uses a scale ranging between 0 and 2 points for the severity of the depressive symptoms. The total score ranges from 0 to 54 , and the higher the calculated score, the more severe the depressive symptoms of the child. The Cronbach's $\alpha$ of the CDI was 0.88 in Cho and Lee study [15].

\section{Revised Children's Manifest Anxiety Scale (RCMAS)}

To evaluate the patients' anxiety, the Revised Children's Manifest Anxiety Scale (RCMAS) was used, which was developed by Reynolds and Richmond and translated and adopted by Choi and Cho [16]. The RCMAS is a self-report scale that consists of a total of 37 questions for children and adolescents from the first grade of elementary school to high school students. It has nine false questions and 28 anxiety questions, which are responded to on a 2-point scale of "yes" and "no." The total score ranges from 0 to 28 , and a higher score indicates greater anxiety. The Cronbach's $\alpha$ was 0.81 in the study by Choi and Cho [16].

\section{Data analysis}

To compare the quality of life reported by the patients and by the caregivers, the independent-samples t-test was used, and Pearson's correlation analysis was applied to analyze the correlation between them. Pearson's correlation analysis was also conducted to examine the correlations between the functional aspects of quality of life and the other factors (i.e., the severity of the symptoms and problems, such as depression and anxiety). To analyze the degree of the influence of each factor on the quality of life, multiple regression analysis was performed. SPSS for Windows version 19 (IBM Corp., Armonk, NY, USA) was used and the level of significance was set at $\mathrm{p}<0.05$.

\section{RESULTS}

\section{Demographic characteristics}

The subjects in the study were recruited between January and February 2017. Sixty-six questionnaires that had appropriate replies for all of the questions were used in the final analysis. The subjects' demographic characteristics are shown in Table 1.

\section{Comparison of the quality of life reported by the patients with the quality of life of the patients reported by the caregivers}

The average of the sum of the PedsQL 4.0 Child Self-Report scores was 78.41 (standard deviation 14.00) while the average of the sum of the PedsQL 4.0 Parent Proxy Report scores was 68.66 (standard deviation 12.98), with the former being significantly higher than the latter (Table 2). When analyzing the sub-factors, the scores for the physical, emotional, and social functions of the PedsQL 4.0 Child Self-Report

Table 1. Demographic characteristics

\begin{tabular}{lc}
\hline & Subject (\%) \\
\hline Gender & $55(83.33)$ \\
Male & $11(16.67)$ \\
Female & $10.68 \pm 2.61$ \\
Age (mean \pm SD) & \\
Comorbidity & $26(39.39)$ \\
ODD or CD & $15(22.73)$ \\
Mood disorder & $16(24.24)$ \\
Anxiety disorder & $17(25.76)$ \\
Tic or Tourette disorder & \\
Number of comorbidities & $18(27.27)$ \\
No comorbidities & $26(39.39)$ \\
1 comorbidities & $22(33.33)$ \\
2 or 3 comorbidities &
\end{tabular}

CD: conduct disorder, ODD: oppositional defiant disorder, SD: standard deviation 
Table 2. Comparisons between PedsQL-Self Report and PedsQL-Parent Proxy Report $(n=66)$

\begin{tabular}{lcccc}
\multicolumn{1}{c}{ PedsQL scale } & PedsQL-self (mean \pm SD $)$ & PedsQL-parent (mean \pm SD) & Mean differences & $P$ \\
\hline Total & $78.41 \pm 14.00$ & $68.66 \pm 12.98$ & 9.75 & $0.000^{*}$ \\
PedsQL somatic & $83.38 \pm 15.17$ & $72.89 \pm 22.19$ & 10.49 & $0.002^{*}$ \\
PedsQL emotional & $77.05 \pm 20.38$ & $65.31 \pm 20.95$ & 11.74 & $0.001^{*}$ \\
PedsQL social & $75.61 \pm 22.98$ & $63.39 \pm 19.27$ & 12.22 & $0.001^{*}$ \\
PedsQL school & $41.96 \pm 40.19$ & $65.00 \pm 14.03$ & -23.05 & $0.000^{*}$ \\
\hline
\end{tabular}

* $p<0.01$. PedsQL: Pediatric Quality of Life Inventory version 4.0 Generic Core Scale, SD: standard deviation

Table 3. Correlation of PedsQL-Self Report and PedsQL-Parent Proxy Report

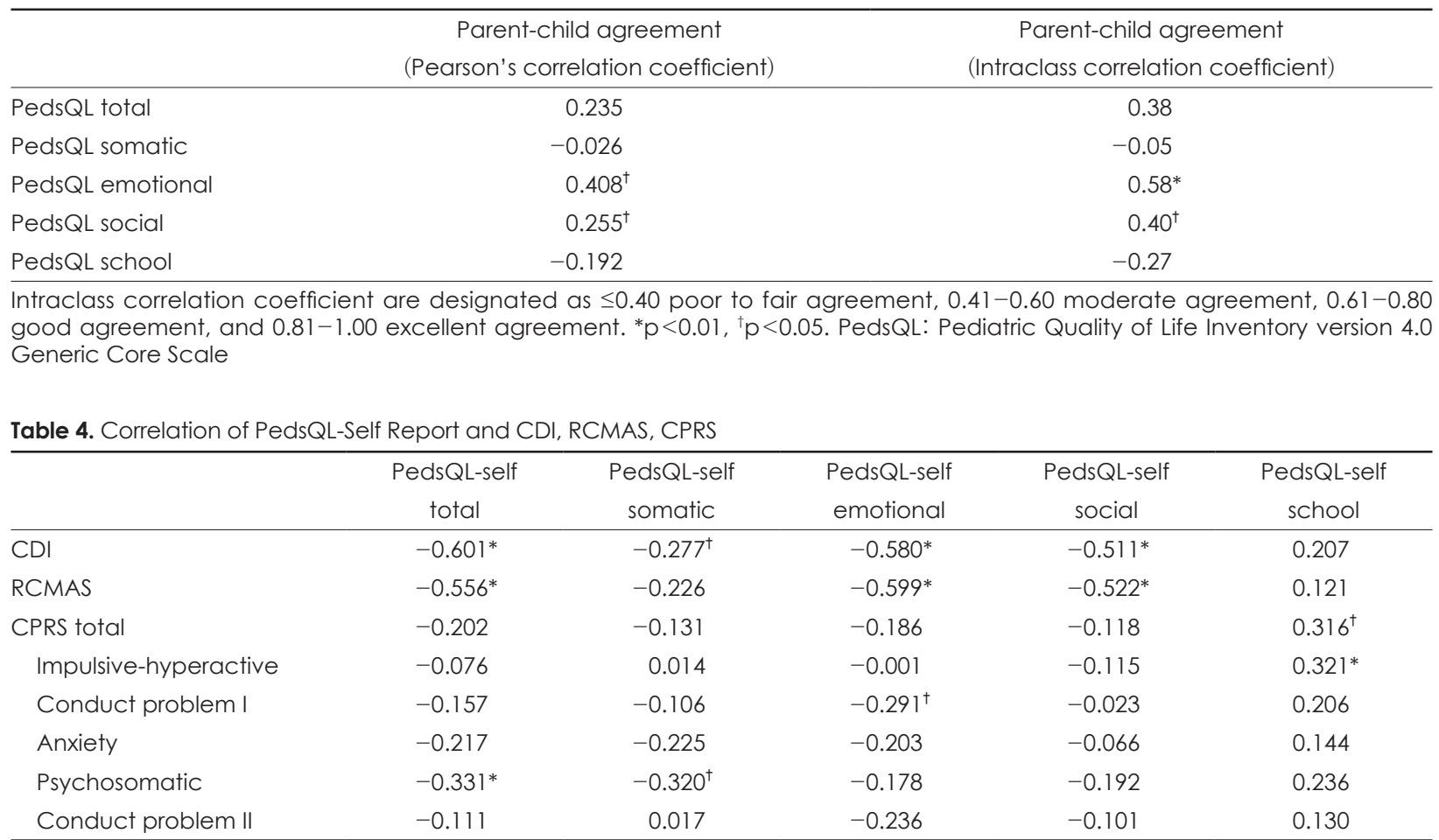

${ }^{*} p<0.01,{ }^{\dagger} p<0.05$. CDI: Children's Depression Inventory, CPRS: Conner's Parent Rating Scale, PedsQL: Pediatric Quality of Life Inventory version 4.0 Generic Core Scale, RCMAS: Revised Child Manifest Anxiety Scale

were significantly higher than those of the PedsQL 4.0 Parent Proxy Report. However, for the school function, the PedsQL 4.0 Child Self-Report score was significantly lower.

\section{Correlation between the quality of life reported by the patients and the quality of life of the patients reported by the caregivers \\ When analyzing the correlation between the total score} and the scores of the sub-factor scales of the PedsQL 4.0 Child Self-Report and PedsQL 4.0 Parent Proxy Report, it was found that the emotional function (Pearson's coefficient=0.408) and social function (Pearson's coefficient $=0.255$ ) were significantly correlated although the association was weak to moderate (Table 3). There were no significant correlations between the other factors.

\section{Correlations between the quality of life reported by the patients and each factor (the severity} of the symptoms, depression, and anxiety)

The total score of the PedsQL 4.0 Child Self-Report was correlated with the scores of the CDI and RCMAS (Pearson's coefficient $=-0.601$ and -0.556 respectively) and there was a correlation with the psychosomatic factor sub-scale of the CPRS (Pearson's coefficient=-0.331) (Table 4). In particular, the CDI was clearly correlated with somatic function (Pearson's coefficient $=-0.277$ ), emotional function (Pearson's coefficient=-0.580), and social function (Pearson's coefficient $=-0.511$ ). In addition, the RCMAS score was significantly correlated with the total score of the PedsQL 4.0 Child SelfReport, emotional function (Pearson's coefficient $=-0.599$ ), and social function (Pearson's coefficient=-0.522). There were no significant correlations between the CPRS and the total 
Table 5. Correlation of PedsQL-Parent Proxy Report and CDI, RCMAS, CPRS

\begin{tabular}{|c|c|c|c|c|c|}
\hline & $\begin{array}{c}\text { PedsQL-parent } \\
\text { total }\end{array}$ & $\begin{array}{c}\text { PedsQL-parent } \\
\text { somatic }\end{array}$ & $\begin{array}{c}\text { PedsQL-parent } \\
\text { emotional }\end{array}$ & $\begin{array}{c}\text { PedsQL-parent } \\
\text { social }\end{array}$ & $\begin{array}{c}\text { PedsQL-parent } \\
\text { school }\end{array}$ \\
\hline CDI & -0.096 & -0.069 & -0.124 & -0.083 & 0.050 \\
\hline RCMAS & -0.066 & -0.078 & -0.152 & -0.121 & 0.086 \\
\hline CPRS total & $-0.431^{*}$ & -0.059 & $-0.542^{*}$ & $-0.418^{*}$ & -0.236 \\
\hline Impulsive-hyperactive & $-0.265^{\dagger}$ & -0.006 & -0.227 & $-0.395^{*}$ & -0.143 \\
\hline Conduct problem I & $-0.367^{*}$ & -0.067 & $-0.540^{*}$ & $-0.296^{\dagger}$ & -0.128 \\
\hline Anxiety & $-0.435^{*}$ & 0.002 & $-0.665^{*}$ & $-0.319^{\dagger}$ & $-0.265^{\dagger}$ \\
\hline Psychosomatic & $-0.317^{\dagger}$ & -0.224 & $-0.369 *$ & -0.062 & $-0.301^{\dagger}$ \\
\hline Conduct problem II & -0.190 & -0.075 & $-0.279^{\dagger}$ & $-0.349^{\dagger}$ & -0.086 \\
\hline
\end{tabular}

${ }^{*} p<0.01,{ }^{\dagger} p<0.05$. CDI: Children's Depression Inventory, CPRS: Conner's Parent Rating Scale, PedsQL: Pediatric Quality of Life Inventory version 4.0 Generic Core Scale, RCMAS: Revised Child Manifest Anxiety Scale

Table 6. Associations of PedsQL (Self Report and Parent Proxy Report) with other variables by multiple regression analysis

\begin{tabular}{|c|c|c|c|c|c|c|}
\hline \multirow{2}{*}{ Variables } & \multicolumn{3}{|c|}{ PedsQL-self } & \multicolumn{3}{|c|}{ PedsQL-parent } \\
\hline & $\beta \pm S E$ & $\mathrm{R}^{2}$ & $\mathrm{p}$ & $\beta \pm S E$ & $\mathrm{R}^{2}$ & $\mathrm{p}$ \\
\hline CDI & $-1.147 \pm 0.196$ & 0.362 & 0.000 & & & \\
\hline CPRS & & & & $-0.398 \pm 0.112$ & 0.174 & 0.001 \\
\hline
\end{tabular}

CDI: Children's Depression Inventory, CPRS: Conner's Parent Rating Scale, PedsQL: Pediatric Quality of Life Inventory version 4.0 Generic Core scale, SE: standard error

score of the PedsQL 4.0 Child Self-Report or the sub-scales in general, although there was a significant correlation between the school function of the PedsQL 4.0 Child Self-Report and the total score of the CPRS (Pearson's coefficient=0.316), particularly for the aspect of impulsivity-hyperactivity (Pearson's coefficient $=0.321$ ).

Correlations between the quality of life reported by the caregivers and each factor (the severity of the symptoms, depression, and anxiety)

The total score of the PedsQL 4.0 Parent Proxy Report was correlated with the total score of the CPRS (Pearson's coefficient=-0.431) and the sub-scales of impulsivity-hyperactivity (Pearson's coefficient=-0.265), conduct problems I (Pearson's coefficient $=-0.367$ ), anxiety (Pearson's coefficient $=-0.435$ ), and psychosomatic factors (Pearson's coefficient $=-0.317$ ) (Table 5). The scores of the emotional and social functions of the PedsQL 4.0 Parent Proxy Report showed clear correlations with most of the sub-scales and the total score of the CPRS.

Level of the influence of each factor (the severity of the symptoms, depression, and anxiety) on the quality of life reported by the patients and that reported by the caregivers

In order to comprehend the practical influence of the severity of the ADHD symptoms and emotional problems, including depression and anxiety, a multiple regression analysis was conducted (Table 6). The CDI, RCMAS, and CPRS scores were used as variables in the analysis. As a result, the total score of the PedsQL 4.0 Child Self-Report was significantly predicted by the CDI score (beta coefficient $=-1.147$, standardized beta coefficient $=-0.603$ ) and the total score of the PedsQL 4.0 Parent Proxy Report was significantly predicted by the CPRS score (beta coefficient $=-0.398$, standardized beta coefficient $=-0.417$ ).

\section{DISCUSSION}

In this study, after obtaining both the ADHD patients' and their caregivers' evaluation of the patients' quality of life and comparing them, the correlations between these factors were examined. The correlations between the quality of life reported by the respective parties and the severity of the ADHD symptoms and the emotional problems, including depression and anxiety, which may affect quality of life, were then investigated. Through this, it was confirmed that the quality of life reported by the patients and that reported by the caregivers was different, and that the evaluation of the quality of life was affected by different and respective factors.

In general, the quality of life reported by the patients was higher than that reported by the caregivers, and it was similar to that reported in previous overseas studies. However, the lower quality of life reported by the patients in terms of school function is different to that found in the previous literature $[17,18]$. These results indicate that domestic patients with ADHD experience worse lowering of quality of life in school than the overseas cases, reflecting the Korean cul- 
tural feature of emphasizing academic attainment. Considering the domestic research's [5] findings that improving academic performance and ability has a more important influence on the improvement of quality of life in patients with $\mathrm{ADHD}$, it is necessary to identify how to explore and improve positive academic performance ability when treating ADHD patients.

In the correlation between the quality of life considered by the patients and that perceived by their caregivers, the emotional and social functions were only weakly to moderately correlated, yet the rest of the functions were weakly to fairly correlated. Even though this is a better result than that found in the previous studies [18], there is a huge gap between the quality of life reported by the patients and that reported by the caregivers. These results confirm that it is important to investigate the quality of life by directly asking the patients, as mentioned in the Introduction.

There were clear correlations between the quality of life and emotional problems, including depression and anxiety, felt by the patients. However, the severity of the symptoms did not show a clear correlation with the comprehensive quality of life reported by the patients, while it was positively correlated with symptoms of impulsivity-hyperactivity. This is different to the findings from previous studies [10] that hyperactivity-impulsivity (i.e., ADHD symptoms) is linked to the quality of life reported by the patients rather than emotional problems. Of course, in this study, both the quality of life and emotional problems were reported by the patients themselves and were correlated, whereas in the study by Becker et al. [10], quality of life was reported by the patients while emotional problems were reported by the caregivers and they were correlated, resulting in their different influences. On the other hand, although the use of medication for symptom control lowers the severity of the symptoms, it may be a sign that the patients' quality of life may be adversely affected by the various side effects of the drugs. Therefore, careful observation of the quality of life is needed when treating ADHD.

In general, the quality of life of the patients reported by the caregivers was more strongly related to the severity of the symptoms rather than to the emotional problems, including depression and anxiety, which is a similar finding to various previous studies [6-8]. In the results of the multiple regression analysis, the most expected factor of quality of life reported by the patients was depression while the most expected factor of quality of life reported by the caregivers was the severity of the symptoms. This result indicates that treating the accompanying symptoms of depression will be more helpful for improving the quality of life felt by the patients themselves when treating ADHD patients, and it is necessary to explore and treat the patients' depressive symptoms in a pos- itive way.

In this study, there were some limitations. First, selection bias may have occurred as the sample was limited to outpatients who consented to participate in the study. Second, the correlations among the questionnaires used in the study may have affected the results. Third, the research subjects were recruited from one hospital, which limits the generalization of the findings. Fourth, the sample size was not sufficient. Fifth, the study did not consider differences depending on the types of drugs used for treatment or comorbidities. Sixth, although the patients were diagnosed with ADHD on an outpatient basis, a detailed diagnostic process and the use of structured diagnostic tools were not confirmed.

\section{CONCLUSION}

This study demonstrates that there are clear differences between the quality of life reported by the patients themselves and their quality of life reported by their caregivers. For these domestic patients, the quality of life in school was far lower, which suggests that it is necessary to investigate how to promote academic performance and improve ability. In addition, in order to increase the comprehensive quality of life reported by the patients, treating the accompanying depressive symptoms is critical. These results indicate the need to positively explore and treat depressive symptoms for ADHD patients in the future.

\section{Conflicts of Interest}

The authors have no financial conflicts of interest.

\section{REFERENCES}

1) Coghill D, Danckaerts M, Sonuga-Barke E, Sergeant J, ADHD European Guidelines Group. Practitioner review: quality of life in child mental health--conceptual challenges and practical choices. J Child Psychology Psychiatry 2009;50:544-561.

2) Danckaerts M, Sonuga-Barke EJ, Banaschewski T, Buitelaar J, Döpfner M, Hollis C, et al. The quality of life of children with attention deficit/hyperactivity disorder: a systematic review. Eur Child Adolesc Psychiatry 2010;19:83-105.

3) Weis M, Weis G. Attention deficit hyperactivity disorder. In: Lewis M, editor. Child and adolescent psychiatry, 3rd ed. Philadelphia: Lippincott Williams \& Wilkins;2002. p.645-670.

4) Johnston C, Mash EJ. Families of children with attention-deficit/ hyperactivity disorder: review and recommendations for future research. Clin Child Fam Psychol Rev 2001;4:183-207.

5) Kim HI, Kim BN, Cho SC, Shin MS, Yoo HJ, Kim JW, et al. Change of quality of life in children with ADHD after 12 weeks OROSmethylphenidate treatment. J Korean Acad Child Adolesc Psychiatry 2012;23:103-108.

6) Riley AW, Spiel G, Coghill D, DÖpfner M, Falissard B, Lorenzo MJ, et al. Factors related to health-related quality of life (HRQoL) among children with ADHD in Europe at entry into treatment. Eur Child Adolesc Psychiatry 2006;15 Suppl 1:I38-I45.

7) Klassen AF, Miller A, Fine S. Health-related quality of life in children and adolescents who have a diagnosis of attention-deficit/hy- 
peractivity disorder. Pediatrics 2004;114:e541-e547.

8) Newcorn JH, Spencer TJ, Biederman J, Milton DR, Michelson D. Atomoxetine treatment in children and adolescents with attentiondeficit/hyperactivity disorder and comorbid oppositional defiant disorder. J Am Acad Child Adolesc Psychiatry 2005;44:240-248.

9) Wehmeier PM, Schacht A, Barkley RA. Social and emotional impairment in children and adolescents with ADHD and the impact on quality of life. J Adolesc Health 2010;46:209-217.

10) Becker A, Roessner V, Breuer D, Döpfner M, Rothenberger A. Relationship between quality of life and psychopathological profile: data from an observational study in children with ADHD. Eur Child Adolesc Psychiatry 2011;20 Suppl 2:S267-S275.

11) Klassen AF. Quality of life of children with attentiondeficit hyperactivity disorder. Expert Rev Pharmacoecon Outcomes Res 2005;5: 95-103.

12) Klassen AF, Miller A, Fine S. Agreement between parentand child report of quality of life in children with attention-deficit/hyperactivity disorder. Child Care Health Dev 2006;32:397-406.

13) Kook SH, Varni JW. Validation of the Korean version of the pediatric quality of life inventory 4.0 (PedsQL) generic core scales in school children and adolescents using the Rasch model. Health Qual Life Outcomes 2008;6:41.

14) Park EH, So YK, Kim YS, Choi NK, Kim SJ, Noh JS, et al. The reliability and validity of Korean Conners Parent and Teacher Rating scale. J Korean Acad Child Adolesc Psychiatry 2003;14:183-196.

15) Cho SC, Lee YS. Development of the Korean form of the Kovacs' Children's Depression Inventory. J Korean Neuropsychiatr Assoc 1990;29:943-956.

16) Choi JS, Cho SC. Reliability and validity of revised children's manifest anxiety scale: assessment of anxiety in children. J Korean Neuropsychiatr Assoc 1990;29:691-702.

17) Bastiaansen D, Koot HM, Bongers IL, Varni JW, Verhulst FC. Measuring quality of life in children referred for psychiatric problems: psychometric properties of the PedsQL 4.0 generic core scales. Qual Life Res 2004;13:489-495.

18) Limbers CA, Ripperger-Suhler J, Heffer RW, Varni JW. Patient-reported Pediatric Quality of Life Inventory ${ }^{\mathrm{TM}} 4.0$ Generic Core Scales in pediatric patients with attention-deficit/hyperactivity disorder and comorbid psychiatric disorders: feasibility, reliability, and validity. Value Health 2011;14:521-530. 